\title{
Transposition
}

Musique et Sciences Sociales

$4 \mid 2014$

Musique et conflits armés après 1945

\section{Conflits armés, idéologie et technologie dans Für Paul Dessau de Luigi Nono}

Armed conflicts, Ideology and Technology in Luigi Nono's Für Paul Dessau

\section{Luis Velasco-Pufleau}

\section{(2) OpenEdition}

\section{Journals}

Édition électronique

URL : http://journals.openedition.org/transposition/1060

DOI : 10.4000/transposition. 1060

ISSN : 2110-6134

Éditeur

CRAL - Centre de recherche sur les arts et le langage

Référence électronique

Luis Velasco-Pufleau, "Conflits armés, idéologie et technologie dans Für Paul Dessau de Luigi Nono », Transposition [En ligne], 4 | 2014, mis en ligne le 15 juillet 2014, consulté le 02 janvier 2020. URL

http://journals.openedition.org/transposition/1060 ; DOI : 10.4000/transposition.1060

Ce document a été généré automatiquement le 2 janvier 2020.

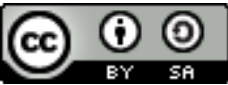

La revue Transposition est mise à disposition selon les termes de la Licence Creative Commons Attribution - Partage dans les Mêmes Conditions 4.0 International. 


\section{Conflits armés, idéologie et technologie dans Für Paul Dessau de Luigi Nono}

Armed conflicts, Ideology and Technology in Luigi Nono's Für Paul Dessau

Luis Velasco-Pufleau

Le conflit décisif est le conflit armé. La révolution bolchevique de 1917, la longue marche chinoise, la Sierra Maestra et le choix révolutionnaire des peuples vietnamien, africains et latinoaméricains nous l'enseignent. Luigi Nono, $1969^{1}$.

\section{Introduction}

1 Au-delà de l'horreur que provoque la guerre en elle-même, certains conflits armés du $\mathrm{XX}^{\mathrm{e}}$ siècle ont suscité un grand espoir chez des artistes engagés dans les luttes politiques internationales de leur époque. Ce fut notamment le cas du compositeur italien Luigi Nono (1924-1990). Pour les intellectuels marxistes comme Nono, les luttes de libération du tiers-monde semblaient annoncer un bouleversement de l'ordre politique à une grande échelle et représentaient la libération du joug colonial, l'indépendance économique et culturelle ainsi qu'une étape nécessaire pour la construction d'une nouvelle société socialiste - un moment décisif au cours duquel les structures socio-économiques et culturelles pouvaient être bouleversées ${ }^{2}$. Dans cette optique, de la Révolution russe à la guerre du Vietnam, en passant par la Révolution cubaine et les guerres de libération (néo)coloniales du tiers-monde durant les années 1960 et 1970, les conflits armés ont été pensés par Nono, dans une perspective internationaliste, comme l'aboutissement de la lutte des classes et comme une occasion pour bâtir une société plus juste et équitable 3 . 
2 L'objectif de cet article est double. D'une part, je propose d'examiner de quelle façon Luigi Nono place certains des conflits armés du $\mathrm{XX}^{\mathrm{e}}$ siècle, dont les luttes d'émancipation du tiers-monde, au cœur de son œuvre pour bande magnétique Für Paul Dessau (1974)4. D'autre part, j'analyserai la relation entre l'utilisation de la technologie par le compositeur, la pensée politique de ce dernier et les dimensions historique et esthétique de cette œuvre - notamment dans le contexte de la Guerre froide et de l'idéologie marxiste. J'apporterai une attention particulière à la contextualisation historique des prises de position de Nono et du matériau utilisé dans Für Paul Dessau afin de mettre en perspective son espoir en l'utopie socialiste et la fonction stratégique que revêtait pour lui la politisation de la musique.

Dernière œuvre de Luigi Nono pour bande magnétique seule ${ }^{5}$, Für Paul Dessau est composée en 1974 dans le Studio di Fonologia de la RAI à Milan - avec l'assistance technique de Marino Zuccheri - et conçue comme un hommage au compositeur allemand Paul Dessau (1894-1979) à l'occasion de son $80^{e}$ anniversaire ${ }^{6}$. Cette œuvre peut être analysée comme une "clairière " dans sa production musicale car elle constitue la synthèse de certains procédés compositionnels, de l'utilisation des nouvelles technologies et des thématiques politiques développées par Nono dans les années qui la précèdent. Cependant, Für Paul Dessau est aussi la dernière d'une longue lignée d'œuvres au contenu expressément politique, et se trouve intimement liée à certaines d'entre elles par le matériau sonore, les textes et les sujets extramusicaux auxquels elle fait référence ${ }^{7}$.

Partant d'une analyse du matériau (sonore et discursif) et des incidences de celui-ci sur la structure formelle de Für Paul Dessau, je m'appuierai sur les écrits du compositeur afin de contextualiser les enjeux idéologiques, politiques et esthétiques présents dans cette œuvre. Comme l'écrivait Nono lui-même en 1969 :

Il est évident que si l'on n'étudie pas et si l'on n'analyse pas ma pratique compositionnelle, y compris dans son rapport entre technique et idéologie, et si l'on reste prisonnier de conceptions traditionnelles, aujourd'hui réactionnaires, on déforme ou méconnait, dans la technique comme dans le moment idéologique qui devient musique, ma position active de musicien totalement engagé dans la lutte politique d'aujourd'hui ${ }^{8}$.

Plus largement, l'ambition de ce texte est de contribuer à la compréhension et à la mise en perspective du marxisme défendu par Luigi Nono en tant qu'intellectuel et artiste militant au Parti Communiste Italien ( $\mathrm{PCI}$ ), dans son articulation avec ses idées esthétiques et son activité de compositeur. Pour cela, je m'appuierai sur trois notions, empruntées à Antonio Gramsci (1891-1937), qui sous-tendent la pensée politique et l'action artistique de Nono: les notions d'idéologie, d'intellectuel et d'hégémonie9. Concernant l'idéologie, elle doit être comprise, d'après Gramsci, comme "une conception du monde qui se manifeste implicitement dans l'art, dans le droit, dans l'activité économique, dans toutes les manifestations de la vie individuelle et collective $^{10}$ ». Pour Nono, l'idéologie devrait servir de guide pour l'action artistique car elle implique une conscience - et trouverait sa source dans la réalité commune au plus grand nombre (le "peuple»). Elle engagerait aussi une éthique qui rendrait inséparable création poétique et réflexion politique, comme il l'écrit en 1966 à propos de la notion d'engagement en musique :

Lorsqu'on parle d'« engagement", en musique, aujourd'hui, c'est souvent sur le plan théorique ou technique, mais rarement sur le plan idéologique. Or, contrairement à ce que croient beaucoup, ces deux formes d'engagement ne sont 
pas inconciliables. En partant de la réalité la plus quotidienne, la plus actuelle, en s'appuyant sur les grands élans de révolte et d'espoir qui secouent notre monde, on peut, hors de tout réalisme infantile, faire œuvre d'imagination qui satisfasse autant l'aide marchante de la pensée contemporaine que les grandes masses. Mais les relations du créateur et des foules (de la classe ouvrière en particulier) ne doivent plus être celles de professeur à élèves, d'initiateur à néophytes. Il faut qu'ils se retrouvent d'abord à l'origine de l'œuvre ${ }^{11}$.

6 Ainsi, pour Nono, le compositeur devrait mettre en forme « avec toutes les ressources que mettent à sa disposition les nouveaux langages sonores et visuels ${ }^{12}$ ", les idées, les données et le matériau issus des luttes politiques et sociales afin de contribuer à la prise de conscience nécessaire à la transformation collective de la société. Ceci implique aussi que les choix du compositeur ne sont jamais neutres et qu'il est responsable, en tant qu'intellectuel organique ${ }^{13}$, des conséquences de ces choix. C'est ainsi que dans son texte «Le pouvoir musical " (Il potere musicale, paru en septembre 1969), Nono, insistant sur sa qualité d'artiste engagé, définit sa position esthétique qui consiste à concevoir « une culture comme moment de prise de conscience, de lutte, de provocation, de discussion et de participation ${ }^{14} »$. Ceci impliquerait pour lui l'utilisation critique des legs du passé mais aussi le refus de toute conception eurocentrique et de toute vision aristocratique de la culture et des langages artistiques. Il affirme que ce qu'il entend par « faire de la musique » est quelque chose qui l'engage « au même titre que de participer à une manifestation, de [se] heurter à la police, ou demain, qui sait, de participer à la lutte armée ${ }^{15}$ ". Il oppose cette conception créatrice à celles d'autres compositeurs. Ainsi, sa critique de la position de Stockhausen nous éclaire de façon explicite sur sa conception éthique et esthétique de l'utilisation de la technologie dans son rapport avec les luttes politiques du tiers-monde. Nono définit la position de l'Allemand de la façon suivante :

la technologie comme valeur, la théorisation d'une évolution technologicoesthétique indolore, la relation naturelle avec les lieux de la production technique la plus avancée, c'est-à-dire les États-Unis et l'Occident, et le mépris aristocratique pour toutes les autres cultures, sans même évoquer le Tiers-Monde ${ }^{16}$.

Luigi Nono qualifie cette position d'impérialiste - quatre ans avant Cornelius Cardew et son livre Stockhausen serves Imperialism - car, selon lui, elle exalterait l'évolution technoscientifique comme seul moment de vérité, sans aucune conscience critique du prix de l'exploitation et du pillage économique qui rend ce développement technique possible ${ }^{17}$. Sa critique vise particulièrement le travail développé par Stockhausen dans Hymnen, où il utilise un grand nombre d'hymnes nationaux et politiques comme matériau de base et dont une seconde version - Hymnen (Dritte Region), pour électronique et grand orchestre - avait été commandée par Leonard Bernstein et l'Orchestre Philharmonique de New York au début de l'année $1969^{18}$. Soucieux de concilier l'activité créative du compositeur avec une éthique et une responsabilité idéologique propres au rôle des intellectuels, Nono se demande alors :

quel sens cela a-t-il alors de parler d'un nouvel espace acoustique, de nouveaux milieux, d'une nouvelle psychologie de l'écoute, de nouvelles techniques, d'un höherer Mensch [d'un homme supérieur] s'ils ne sont pas rapportés à de nouvelles structures sociales et humaines qui ne se basent pas sur l'exploitation et la domination néo-capitaliste et néo-colonialiste, en un mot: sur des structures socialistes ou qui tendent vers le socialisme ${ }^{19}$ ?

8 En effet, les compositeurs - en tant qu'intellectuels organiques - ont pour Nono la fonction hégémonique de lutter contre la conception du monde de la classe dominante 
(bourgeoisie) par la création musicale et par la promotion d'un imaginaire révolutionnaire et socialiste ${ }^{20}$. Cette action doit prendre en compte les conditions sociales, économiques et culturelles de leur activité créative ainsi que ses conséquences. Comme le raconte Helmut Lachenmann, «Luigi Nono n'a cessé de se solidariser avec l'agitation sur cette terre, il l'a faite sienne. C'est en puisant dans cette agitation que son activité artistique et sa nature humaine se sont constamment renouvelées ${ }^{21} »$.

\section{Für Paul Dessau : synthèse, espace et polyphonie sonore}

Depuis 1960 et jusqu'à la moitié des années 1970, l'utilisation que fait Luigi Nono de nouveaux outils technologiques au Studio di Fonologia de la RAI de Milan s'inscrit au sein du projet esthétique et politique consistant à placer son œuvre, et par conséquent les moyens utilisés pour la produire, au service de la lutte de classes et de la justice sociale. Comme l'affirme Laurent Feneyrou, Nono « considère que la transformation des modes de communication et de dialogue avec le monde s'applique au langage musical de la même manière qu'aux formes de la société22 ». Ainsi, comme le déclare Nono en 1969, la maitrise de la technique et le travail au sein du studio électronique deviennent indispensables pour la diffusion des idées nécessaires à l'hégémonie culturell ${ }^{23}$ et idéologique des forces révolutionnaires :

Il nous faut comprendre et nous approprier tout élément et toute conquête technique effectivement novatrice, que nous différencions et que nous responsabilisons par notre conception théorique et pratique de la lutte actuelle, et que nous associons alors à notre capacité d'invention et de création pour l'hégémonie, selon le terme de Gramsci, des forces révolutionnaires, dans leur pratiques destructrices, constructives et intellectuelles. Un exemple: le développement et l'application de la technique électronique dans la musique contemporaine, le studio électronique. C'est une conquête et une possibilité expressive inédite pour la création musicale ${ }^{24}$.

Parmi les possibilités expressives inédites offertes par l'électronique à la création musicale de Nono, se trouve la possibilité d'utiliser comme source un matériau figé préalablement par l'enregistrement, de modifier sa morphologie et de l'insérer dans un nouvel espace acoustique. Ainsi, Für Paul Dessau est constituée de deux catégories de matériaux complémentaires, qui peuvent être classés selon leur provenance acoustique et leurs fonctions formelles. D'une part, nous trouvons les voix d'hommes politiques Vladimir I. Lénine, Ernst Thälmann, Patrice Lumumba, Ernesto « Che » Guevara et Fidel Castro - enregistrées lors de discours historiques entre 1919 et 1963 et accessibles à Nono sous la forme de disques vinyles ${ }^{25}$. Ces discours hautement symboliques dans l'histoire du socialisme et du tiers-mondisme renvoient aux luttes de libération auxquelles Nono s'identifiait lui-même, tout comme son ami Paul Dessau ${ }^{26}$, s'inscrivant de ce fait «dans l'histoire des mouvement révolutionnaires, qu'il avait étudiée et à laquelle il se savait lié27 ». Comme l'explique Nono à propos de son œuvre :

Dans cette manifestation de solidarité à l'écoute de Paul Dessau, j'ai pris pour thème de cette composition notre lutte commune internationale. Des mots de Lénine, extraits de Qu'est-ce que le pouvoir des Soviets? et de l'Adresse à l'armée rouge, des mots de Thälmann, un enregistrement illégal de Lumumba dans sa cellule, avant son assassinat, des mots d'Ernesto Che Guevara sur trois années de guerre au Viêt-Nam et de la Deuxième Déclaration de La Havane de Fidel Castro déterminent ma 
structure musicale. Cette structure est ainsi construite pour libérer les forces de l'art et du combat, à travers la synthèse de ces mots de notre temps, lesquels rapprochent de notre réalité le combat et l'art, et leur confèrent une nouvelle dimension. Genossen Paul Dessau. Camarade de Berlin en RDA ${ }^{28}$.

11 D'autre part, Nono utilise des extraits d'enregistrements de trois de ses propres œuvres afin de soutenir et d'encadrer dramatiquement les discours politiques : Il canto sospeso (1955-1956, pour soprano, contralto, ténor et orchestre) ; Non consumiamo Marx (1969, pour bande magnétique $\left.{ }^{29}\right)$; ainsi que Como una ola de fuerza y luz (1971-1972, pour soprano, piano, orchestre et bande magnétique). Se rapportant aux sujets politiques abordés par ces œuvres, ce matériau crée un système autoréférentiel qui élargit considérablement la portée symbolique de Für Paul Dessau. Tout d'abord, Il canto sospeso, basée sur des lettres de condamnés à mort de la Résistance européenne, crée un lien avec la lutte antifasciste durant et après la Deuxième Guerre mondiale. Cette œuvre avait une grande importance pour Nono et il se remémorait ainsi les années de sa conception et de sa création ${ }^{30}$ :

C'étaient les années de la grande redécouverte des écrits d'Antonio Gramsci, de la brutale répression scelbienne, $\mathrm{du}$ front antifasciste et de la présence continue et discutée de la Resistenza. [...] Années de grandes agitations, luttes polémiques, politiques et culturelles. Que nous ressentions peut-être plus et auxquelles nous participions, en Italie. Et qui exigeaient une rigueur de préparation, d'étude ouverte et innovante, et d'analyse sur le réel, contre les modèles et les dogmatismes en usage, de choix et de conflits souvent violents ${ }^{31}$.

Comme l'écrit Laurent Feneyrou, «dans l'œuvre de Luigi Nono, ce qui, avec Il Canto sospeso, est une dénonciation des crimes commis par le nazisme et le fascisme, deviendra, au cours des années soixante, dès Intolleranza 1960, condamnation du type de société qui conduit aux hitlérismes d'hier et d'aujourd'hui ${ }^{32}$ ». Ainsi, Non consumiamo Marx, composée notamment à partir d'enregistrements des manifestations contre la Biennale de Venise en juin 1968 et des slogans utilisés lors des événements parisiens de mai 68, est conçue comme un manifeste contre les dérives culturelles, sociales et économiques du capitalisme. Nono dédie cette œuvre "à Carlos Franqui, poète révolutionnaire cubain » et parle ainsi des « matériaux de documentation » qu'il utilise dans celle-ci :

Pris dans la rue: Venise, juin 1968. Boycott et lutte des étudiants, des ouvriers et des intellectuels contre la Biennale. Institution culturelle commerciale, acquise aux intérêts des monopoles (Montedison et Ciga) et du pouvoir gouvernemental. Enregistrements effectués par le camarade vénitien Penso.

Mural-politique: 20 slogans relevés sur les murs de Paris (mai 1968) - lutte contre l'État capitaliste et le pouvoir personnel (de Gaulle).

- Situations humaines de la lutte des classes de notre temps, offrant de nouvelles perspectives, une nouvelle signification et une nouvelle praxis au mouvement amoureux-pavésien (Embrasse ton amour sans laisser ton fusil)

- Technique de composition électronique basée sur des voix (enregistrées, élaborées, composées ou en direct) et sur un matériau électronique original ${ }^{33}$.

13 Enfin, Como una ola de fuerza y luz est dédiée à la mémoire de Luciano Cruz, leader du MIR (Mouvement de la gauche révolutionnaire ${ }^{34}$ ) au Chili, mort en septembre 1971 et dont Nono avait fait la connaissance à Santiago durant l'été de la même année. Nono écrit à propos de Cruz et de la genèse de son œuvre :

Son intelligence et son extraordinaire capacité marxiste de combattant pour la liberté chilienne suscitèrent mon admiration et une amitié immédiate entre nous. En septembre 1971 me parvint la nouvelle de sa mort accidentelle et étrange, à l'âge 
de vingt-sept ans seulement. C'est la motivation de cette musique. [...] Sa présenceabsence détermine le choix définitif de la structure sonore, de ses motivations. J'élargis le premier projet en ajoutant une partie vocale (soprano) sur quelques vers dédiés à Luciano Cruz par un poète argentin Julio Huasi, ami de Luciano, et dont j'avais aussi fait la connaissance à Santiago ${ }^{35}$. grande importance pour Nono: le phénomène de la Resistenza européenne, les manifestations internationales de 1968 et la lutte révolutionnaire dans le Chili du front populaire de Salvador Allende. Ces œuvres et les événements qu'elles portent se trouvent à la base de Für Paul Dessau, structurant et soutenant la dramaturgie des discours historiques et élargissant en même temps leur portée symbolique.

\section{Dramaturgie, organisation formelle et principaux procédés compositionnels}

Sur le plan temporel, Für Paul Dessau est structurée par deux types de sections : des Sections Discursives (SD), basées principalement sur l'articulation des discours politiques (soutenus par des fragments des œuvres de Nono) et des Sections Non Discursives (SND), basées exclusivement sur les œuvres de Nono - une introduction et une coda aux extrémités et des Interludes (I) entre chacune des SD (voir tableau et exemple ci-dessous).

Tableau 1. Utilisation des discours politiques et des fragments d'œuvres dans Für Paul Dessau

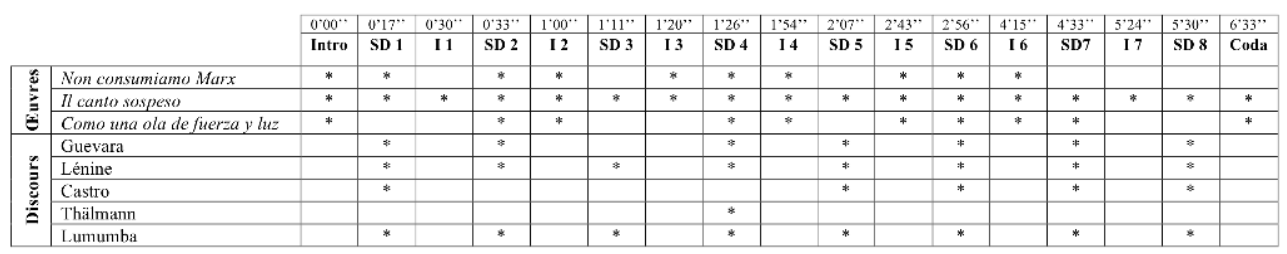

16 La fonction formelle des I est de structurer les SD afin de mieux maîtriser la dramaturgie de leur contenu sémantique. Les fragments des œuvres sont choisis par Nono en fonction de leur force dramatique et agencés la plupart du temps de façon polyphonique, allant d'une nuance $p p$ quand il s'agit d'accompagner et de soutenir les discours (certains fragments des œuvres assurent la continuité entre les I et les SD) à une nuance ff lors des SND (l'introduction, les interludes et la coda). De même, le choix des accompagnements sonores des SD assure une progression dramatique - et non plus seulement sémantique - fondée sur la couleur du son et sur des textures polyphoniques. 
Exemple 1. Sonagramme du début de Für Paul Dessau (0'00"-1'46") : distribution des œuvres et des discours lors de l'introduction, les quatre premières SD et les trois premiers I

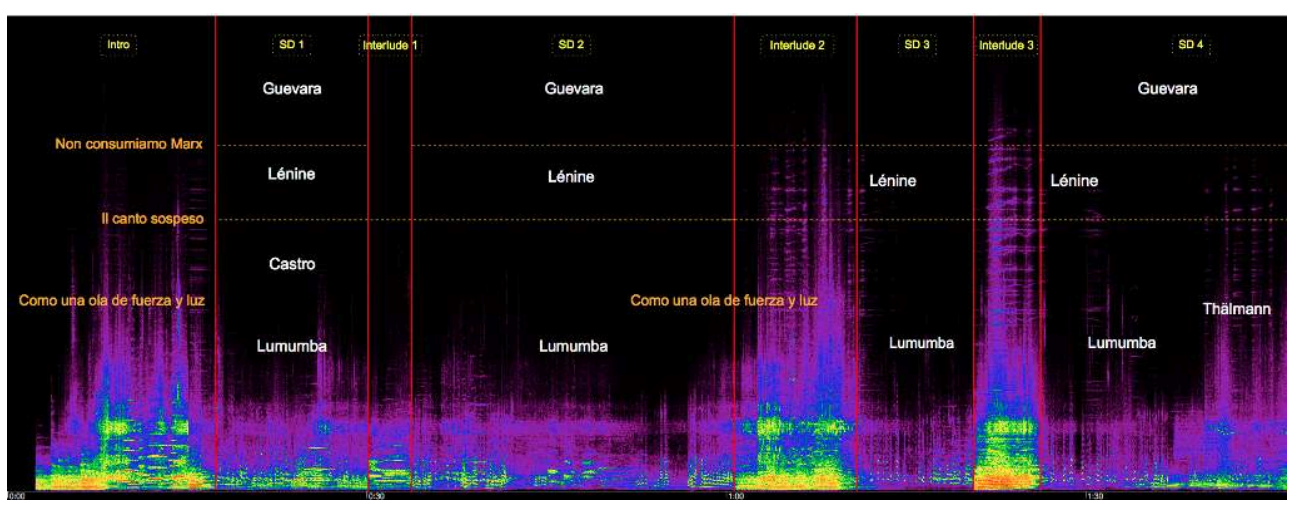

Sonagramme réalisé avec le logiciel d'analyse EAnalysis (http://logiciels.pierrecouprie.fr/? page_id=402).

17 Cette progression dramatique est menée également par les procédés compositionnels utilisés par Nono dans chaque type de section. D'une part, dans les SD, le mixage des voix par stratification privilégie une perception contrapuntique qui met en valeur aussi bien la portée sémantique de certains mots ou phrases que la dimension rythmique et sonore de chaque langue. N'étant pas transformées par des filtres ou des transpositions, les voix sont articulées de façon à ce que le contenu des discours soit compréhensible par l'auditeur. De même, la plupart des discours étant mixés en superposition fade-in et fade-out (fondu-enchaîné), il s'agit plus pour Nono de gérer la dramaturgie du flux sonore que de composer avec des fragments ou des syllabes des discours en question. Sur ce point, il est significatif de souligner que Nono n'utilise pas la fragmentation ou la répétition des mots comme principe de composition dans les SD car il n'expose jamais deux fois la même phrase.

D'autre part, dans les SND, les fragments des œuvres sont montés par juxtaposition (articulation par l'assemblage plus ou moins abrupt des objets sonores) ou par superposition progressive (accumulation ou chevauchement des différentes strates sonores), créant des séquences dont les timbres et profils dynamiques sont nouveaux. Contrairement aux discours, la morphologie et l'espace de la plupart de ces fragments sont transformés par l'ajout en studio de réverbération ou par l'utilisation des filtrages divers, des variations de vitesse et des transpositions.

Étant donné qu'une analyse exhaustive des différentes sections de l'œuvre à la lumière des procédés compositionnels utilisés par Nono me ferait dépasser les dimensions fixées à ce texte, j'analyserai des aspects spécifiques de l'introduction et de la conclusion afin d'illustrer mes propos. Tout d'abord, l'introduction (0'00"-0'17") est composée par la juxtaposition et la superposition d'éléments hétérogènes issus de trois œuvres : sons du piano transposés et voix transformée avec réverbération (Como una ola de fuerza y luz) ; extraits instrumentaux transposés (Il canto sospeso); rumeurs et cris des manifestations (Non consumiamo Marx). Le montage de ces éléments crée un objet sonore avec un nouveau profil dynamique qui remplit la fonction d'ouverture de l'œuvre. 
Exemple 2. Sonagramme de l'introduction de Für Paul Dessau (0'00"-0'17")

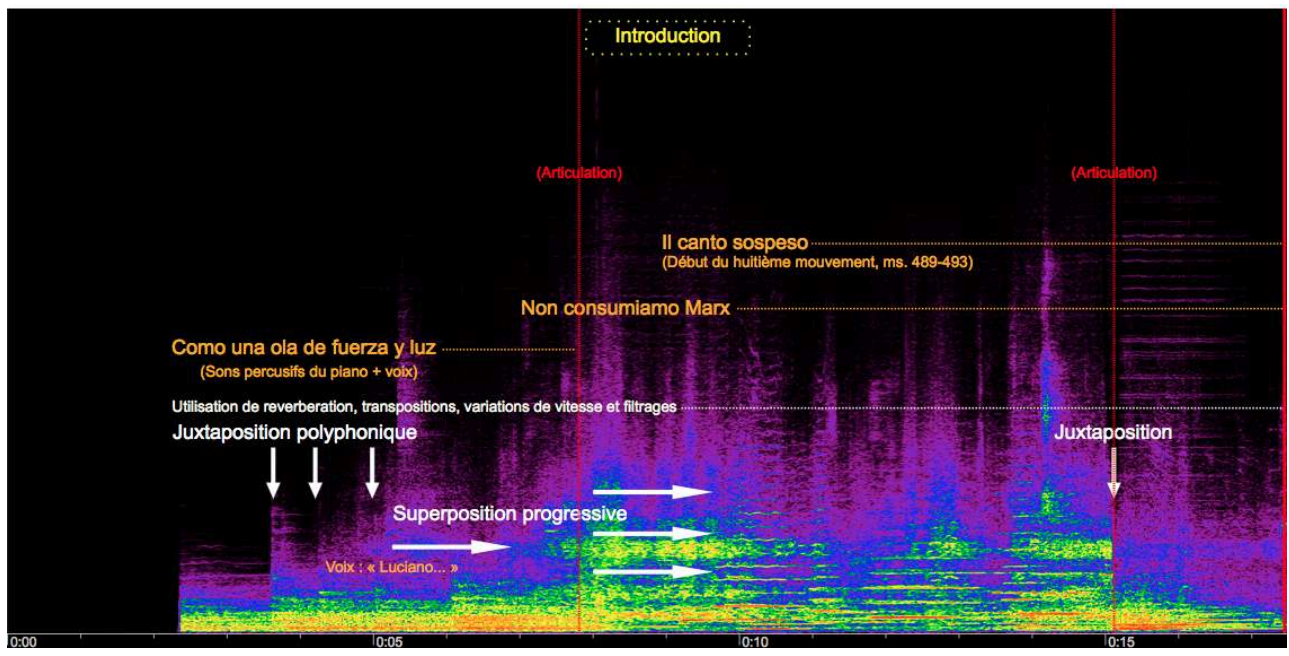

Ensuite, en ce qui concerne la coda (6'33'-6'57'), Nono utilise des sons de piano extraits de Como una ola de fuerza y luz ainsi que divers fragments de Il canto sospeso. Le choix du fragment qui clôt l'œuvre est symboliquement fort : Nono utilise la première phrase de l'intervention chantée de la soprano solo dans le septième mouvement de Il canto sospeso. Il s'agit en effet de la fin de la lettre d'une jeune ukrainienne (Ljuba Sevcova), membre de la résistance dans la ville minière de Krasnodon, à destination de sa mère. Sachant qu'elle sera assassinée par les forces d'occupation nazies, elle lui écrit : «... adieu, maman, ta fille Ljubka s'en va dans la terre humide... ${ }^{36}$ " (voir exemple cidessous).

Exemple 3. Réduction du fragment du septième mouvement de I/ canto sospeso (mesures 429-437) utilisé dans la coda de Für Paul Dessau (6'33"-6'57")

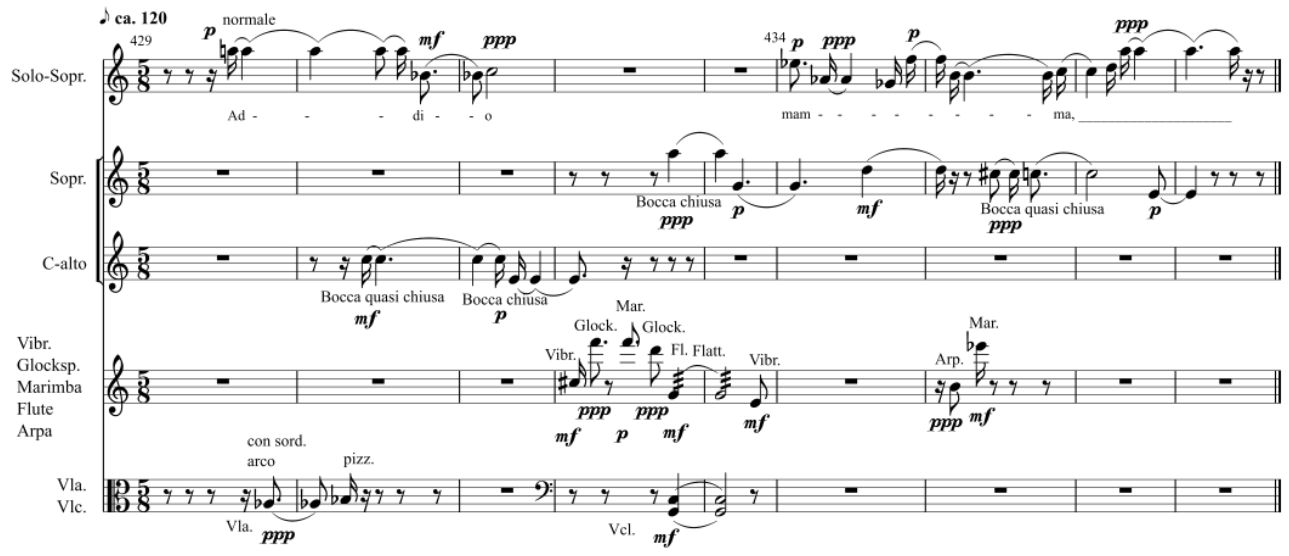

La volonté de Nono de terminer Für Paul Dessau par ce fragment émouvant de Il canto sospeso - qui constitue le fil conducteur sonore tout au long de l'œuvre - peut être interprétée à la lumière de la signification qu'il donnait au phénomène de la Resistenza, qui revêtait pour lui une actualité qui allait bien au delà du moment du conflit armé :

La Resistenza, acte concrètement révolutionnaire et fondamental de notre vie, nécessite, provoque et engendre des choix précis et une conscience novatrice : ni exclusivement, ni une fois pour toutes, lors de la lutte armée, mais dans sa continuité complexe, si nécessaire, constructive et déterminante. [...] Cette Resistenza ne se limite pas à un drapeau glorieux du passé, mais c'est une lutte 
incessante et une conscience nouvelle en développement continu pour l'action subjective et, dans ses intentions, sur le processus objectif qui résulte des principes et des idéaux pour lesquels beaucoup ont déjà donné leur vie ou sont encore assassinés aujourd'hui ${ }^{37}$.

Pour Nono, le musicien participe activement à cette lutte, en la prolongeant dans le présent par sa contribution originale et créative, «par ses études, ses recherches et ses expériences, ainsi que par son imagination inventive; et parce qu'il dialectise les idées et les moyens technico-linguistiques, tous deux dans leurs exigences et leurs propriétés particulières ${ }^{38}$ ». C'est pourquoi, pour Nono, il ne faut pas «limiter le thème de la Resistenza en musique à l'utilisation de textes et de situations de la lutte des partisans, en lui assignant donc une époque déterminée dans l'histoire ». Il faudrait au contraire rechercher l'héritage de la Resistenza « potentiellement présent dans ces expressions où la vérité et la nouveauté de la recherche, de l'invention et de la réalisation élargissent et développent la capacité de l'imagination, l'intelligence de la réception et la conscience de l'homme tendu vers l'élimination des différents garrots de la société néocapitaliste, pour la libération socialiste ${ }^{39} »$. C'est ainsi que la portée politique du travail créatif du compositeur actualise l'héritage des luttes du passé en le rendant visible dans les luttes du présent, ce qui constitue l'un des principaux enjeux de Für Paul Dessau.

Sur le plan spatial, l'œuvre est organisée de façon stéréophonique ${ }^{40}$, Nono ayant placé au centre ses propres œuvres, sur le canal gauche les voix de Lénine et de Guevara et sur le canal droit celles de Thälmann, de Lumumba et de Castro (voir tableau cidessous). Une possible interprétation de cette distribution spatiale pointerait vers la volonté du compositeur de "situer" géographiquement, de "territorialiser " ces œuvres et ces discours, tout en les faisant dialoguer grâce à une articulation polyphonique. Cette analogie entre espace acoustique de l'œuvre et espace géographique servirait à relier dans le moment présent les luttes d'émancipation auxquelles font référence les sons et les discours.

Tableau 2. Disposition spatiale des œuvres et des discours utilisés dans Für Paul Dessau

\begin{tabular}{|c|c|c|}
\hline Canal gauche & Centre & Canal droit \\
\hline $\begin{array}{l}\text { Lénine : } \\
\text { "Qu'est-ce que le pouvoir des Sovicts?» (1919) } \\
\text { "Adresse à l'armée rouge » (1919) }\end{array}$ & $\begin{array}{l}\text { Il canto sospeso }(1955-1956) \\
\text { Non consumiamo Marx (1969) }\end{array}$ & $\begin{array}{r}\text { Ernst Thälmann : } \\
\text { Discours prononcé à Moscou dans le cadre du } \\
\text { Congrès des travailleurs de la métallurgie } \\
\text { (15 février 1928) }\end{array}$ \\
\hline $\begin{array}{l}\text { Ernesto « Che » Guevara : } \\
\text { « Solidarité avec le Sud Vietnam » (1963) }\end{array}$ & Como una ola de fuerza y luz (1971-1972) & $\begin{array}{r}\text { Patrice Lumumba : } \\
\text { «Dernier discours de Patrice Lumumba » (1960) } \\
\text { Fidel Castro: } \\
\text { «Deuxième déclaration de La Havane » (1962) }\end{array}$ \\
\hline
\end{tabular}

En effet, les principaux pays où se mènent les luttes révolutionnaires évoquées dans les discours utilisés se situent sur trois continents : l'Asie (Russie ${ }^{41}$ et Vietnam), l'Afrique (Congo) et l'Amérique latine (Cuba, Chili) - les continents représentés en 1966 dans la conférence Tricontinentale de La Havane ${ }^{42}$. Étant donné que les œuvres de Nono font référence à des faits historiques qui ont eu lieu en Europe (Il canto sospeso, Non consumiamo Marx) et en Amérique Latine (Como una ola de fuerza y luz), le choix de les placer au centre de l'espace sonore leur donnerait une fonction unificatrice par rapport aux luttes énoncées dans les discours. Cette organisation spatiale relie ainsi les luttes "périphériques" à l'héritage et l'expérience de Nono lui-même et esquisse simultanément un nouveau centre possible pour le mouvement socialiste international 
dans le contexte de la Guerre froide : l'Amérique Latine en général et le Chili d'Allende en particulier. Il faut rappeler que Nono était particulièrement attaché au projet socialiste de Salvador Allende et donnait à celui-ci un rôle prépondérant dans le mouvement socialiste mondial - comme il le fit savoir au président Allende dans un télégramme daté du 26 janvier 1973, quelques mois seulement avant le coup d'État (11 septembre 1973) qui renverse le régime démocratique chilien ${ }^{43}$. De même, comme l'écrit Erika Schaller, au début du projet compositionnel de Für Paul Dessau, Nono prévoyait de donner une place centrale à la question du socialisme au Chili et il donne à l'œuvre le titre provisoire de Da Lenin ad Allende (un montaggio) ${ }^{44}$.

\section{La fonction idéologique de l'œuvre}

La fonction référentielle des œuvres de Nono ancre les discours historiques dans l'univers sonore du compositeur et dans le contexte politique précis de la Guerre froide. Mais la dimension sémantique et historique des discours est fondamentale, les mots étant articulés pour être compris par l'auditeur et organisés de façon polyphonique afin de créer un nouveau discours de synthèse revêtant une fonction idéologique précise : relier des causes, désigner des ennemis communs et indiquer les objectifs à atteindre.

À la manière d'un cantus firmus, les deux discours de Lénine ${ }^{45}$ - les plus présents dans l'œuvre, avec celui de Guevara sur la guerre du Vietnam - ont une fonction structurante dans la polyphonie globale. Enregistrés en 1919 durant la guerre civile qui suit la Révolution d'Octobre, ils ont la fonction de motiver les troupes de l'Armée rouge et d'expliquer la visée révolutionnaire du nouveau pouvoir donné aux soviets. Pour Lénine, ce pouvoir

permet à ceux qui étaient opprimés de se relever et de prendre eux-mêmes en main de plus en plus toute la direction de l'État, toute la direction de l'économie, toute la direction de la production. Le chemin des Soviets est le chemin du socialisme, découvert par les masses laborieuses, donc un chemin sûr, donc un chemin invincible ${ }^{46}$.

La fin de son appel à l'armée exalte la victoire du socialisme proclamé par Lénine et souligne en même temps le caractère internationaliste de la lutte du peuple russe : "Camarades soldats de l'Armée Rouge! Tenez bon, tenez ferme, serrez les rangs! Marchez hardiment contre l'ennemi! Nous aurons la victoire. Le pouvoir des propriétaires fonciers et des capitalistes, brisé en Russie, sera vaincu dans le monde entier ${ }^{47}$ ! ». Le discours de Lénine est contrepointé par le discours du «Che » Guevara, qui exalte la lutte armée du peuple vietnamien face à l'agression impérialiste des ÉtatsUnis, reliant symboliquement la Révolution d'Octobre à la guerre du Vietnam. Cette généalogie est exposée par Nono dans une intervention contre la guerre du Vietnam datant de 1966 :

La conquête de la paix pour le Viêt-Nam et son droit [à décider de son destin socialiste] doivent être une nouvelle conquête de tout le mouvement ouvrier et anti-impérialiste, et pour tout ce mouvement, sur la voie ouverte par l'Octobre rouge, par la Longue Marche et par la Sierra Maestra [et de la reprise et de l'accomplissement de notre résistance, pour un monde nouveau $]^{48}$. 
Image 1. Couverture et détail du disque vinyle du discours d'Ernesto « Che » Guevara (prononcé à La Havane, le 20 décembre 1963)

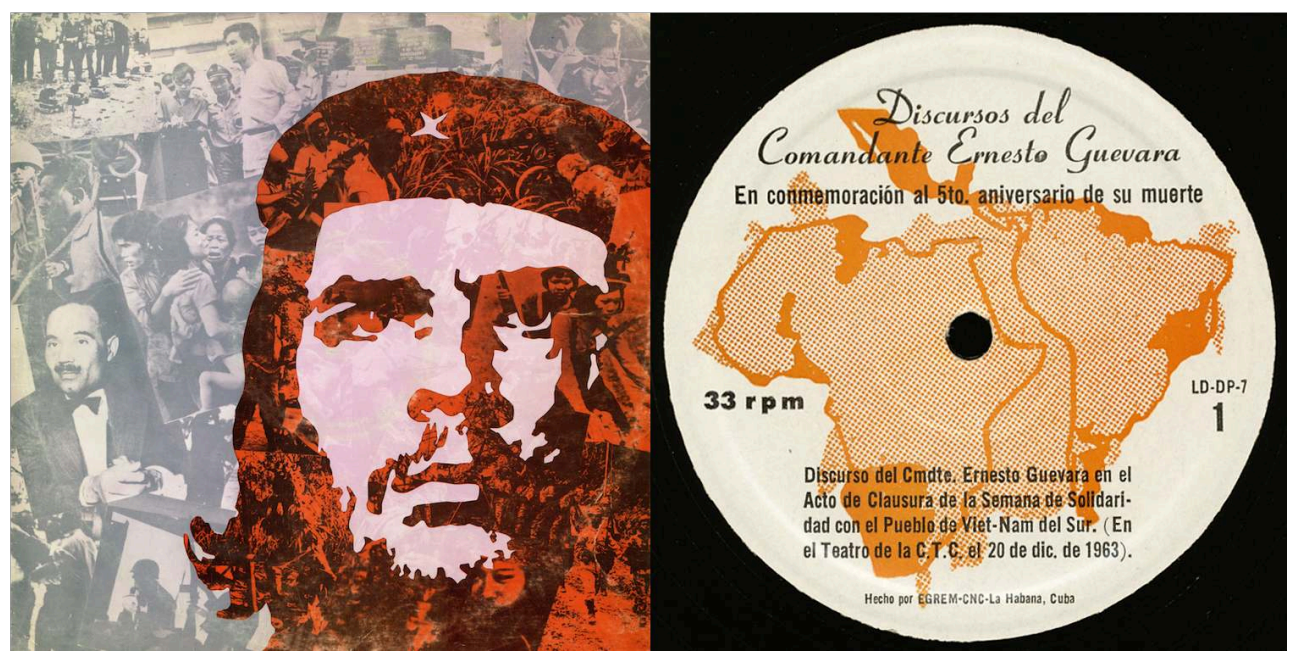

Le discours de Guevara incrimine directement la politique impérialiste menée par les États-Unis et dessine une géographie tricontinentale des résistances tiers-mondistes : "L'Amérique, l'Asie et l'Afrique, les trois continents opprimés commençaient à montrer qu'ils n'accepteraient plus longtemps la présence des pouvoirs coloniaux ${ }^{49} »$. Guevara identifie la lutte armée du peuple vietnamien à la Révolution cubaine et inventorie dans son discours un certain nombre de foyers révolutionnaires et de "guerres de libération", aussi bien en Amérique Latine (Nicaragua, Honduras, Guatemala, Saint Domingue, Colombie, Venezuela et Paraguay), qu'en Asie (Laos, Vietnam) et en Afrique (Angola, Guinée portugaise [Guinée-Bissau]).

Pour sa part, le discours de Fidel Castro intensifie avec un lyrisme et une rhétorique caractéristiques de la Guerre froide les promesses de liberté formulées par ces mouvements de libération, affirmant qu' «il faudra compter avec les pauvres d'Amérique, avec les exploités et méprisés de l'Amérique Latine qui ont décidé d'écrire eux-mêmes, pour toujours, leur propre histoire ${ }^{50}$ ». De même, il prolonge la critique contre les États-Unis établissant un parallèle entre les défauts supposés de ceux-ci et les vertus revendiquées pour Cuba par les acteurs issus de la révolution :

Cuba, pour les alphabétiseurs assassinés ; les États-Unis, pour les assassins

Cuba, pour la vérité ; les États-Unis, pour le mensonge

Cuba, pour la libération ; les États-Unis, pour l'oppression

Cuba, pour l'avenir lumineux de l'humanité; les États-Unis, pour le passé sans espoir

Cuba, pour la paix entre les peuples ; les États-Unis, pour l'agression et la guerre

Cuba, pour le socialisme ; les États-Unis, pour le capitalisme ${ }^{51}$. 
Image 1. Couverture et détail du disque vinyle du discours de Fidel Castro (prononcé à La Havane, le 4 février 1962)

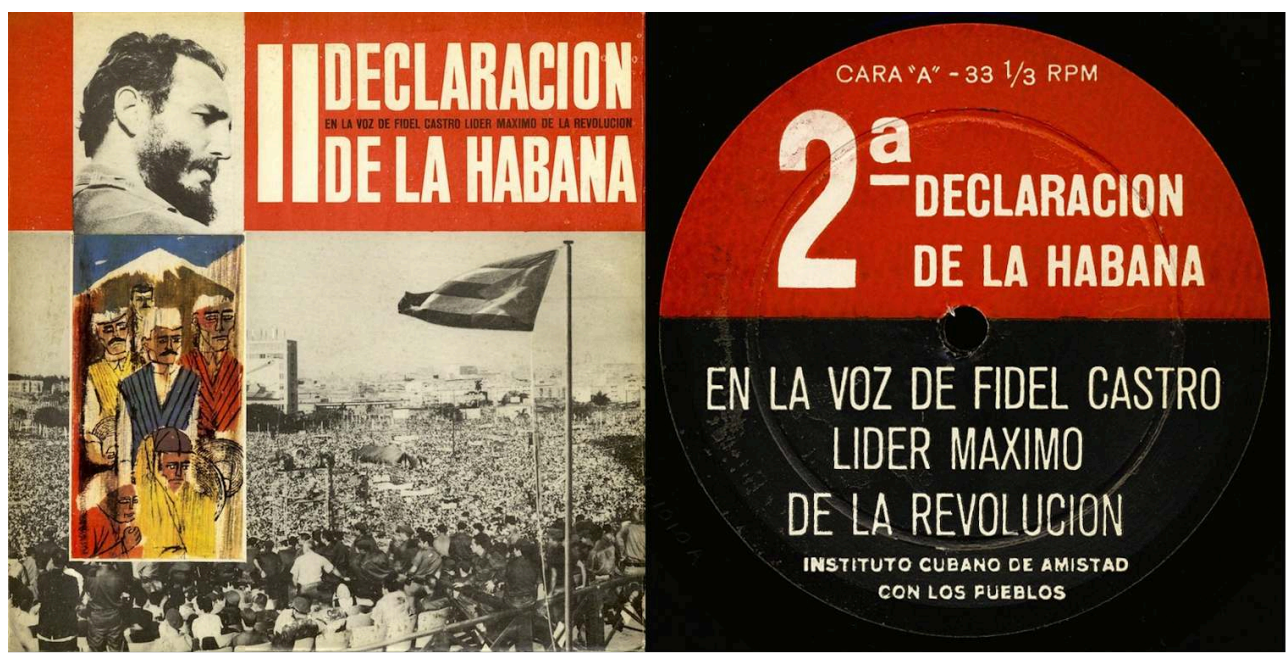

Ainsi que l'exprime Guevara dans son discours, pour Luigi Nono, l'agression militaire des États-Unis «n'est pas seulement contre le Viêt-Nam, mais contre le monde socialiste et contre les mouvements de libération nationaux ${ }^{52} »$. Cela explique la présence dans Für Paul Dessau du discours de Patrice Lumumba, figure emblématique de la lutte d'indépendance du Congo belge et premier ministre (de juin à septembre 1960) de la nouvelle République du Congo ${ }^{53}$. Nono utilise des extraits de ce qui est considéré comme le dernier discours ${ }^{54}$ de ce leader de la décolonisation africaine, mettant en garde contre les diverses formes du néocolonialisme mis en place par les anciennes puissances: "puisqu'on vient de tomber dans un néo-colonialisme, qui est aussi dangereux que le colonialisme que nous venons d'enterrer le 30 juin dernier ${ }^{55}$ », affirme Lumumba dans son discours. Enfin, le discours d'Ernst Thälmann ${ }^{56}$, symbole international de la résistance antifasciste allemande et héros national en Allemagne de l'Est (RDA), appelle à « répondre aux exigences des principales tâches pour la victoire de la révolution prolétarienne ". Sachant que son œuvre serait créée dans l'acte d'hommage à Paul Dessau, et en présence de celui-ci, Nono le salue à travers les mots de Thälmann, qui transmet à son auditoire ses «salutations fraternelles et révolutionnaires ${ }^{57}$ ».

\section{La fonction hégémonique de l'œuvre}

31 Le discours sonore crée par Nono dans Für Paul Dessau - entendu ici comme l'union et l'articulation d'éléments sonores hétérogènes avec une certaine dimension sémantique - est organisé de façon à créer une dramaturgie politique guidée par une idéologie précise. La présence dans l'œuvre des luttes internationales anti-impérialistes contribue à l'hégémonie intellectuelle du socialisme tiers-mondiste défendu par Nono, un socialisme qui puiserait notamment sa force dans l'héritage de Lénine et de Gramsci, tout en respectant les diversités politiques, économiques et culturelles des différents peuples :

La réalisation du socialisme vers lequel nous tendions - écrit Nono en 1971 au directeur du journal $L^{\prime} U n i t \grave{a}^{58}$-, le créant à l'image de notre réalité, inspiré de Lénine et de Gramsci, du soviétisme qui a conduit aux luttes de libération en Asie [...], en Afrique et dans une partie de l'Amérique Latine, que nous construisons avec 
la lutte des travailleurs, des paysans, des ouvriers, des étudiants ; le socialisme pour lequel luttent les travailleurs de la région Baltique, ceux de Detroit et du mouvement Black Panthers dans le bastion de l'impérialisme ${ }^{59}$. socialisme (théorie) de son militantisme politique et de son activité artistique en tant que compositeur et pédagogue (praxis). Suivant l'exemple de Gramsci, Fanon ou Guevara, Nono réfléchit à l'adéquation de la théorie marxiste, de la stratégie révolutionnaire et de la création artistique aux différents contextes historiques et culturels, comme il l'affirme à propos de sa participation en décembre 1971 à un cours latino-américain de musique contemporaine en Uruguay :

Mon séminaire de composition se déroula à la lumière des indications culturelles et politiques d'Antonio Gramsci, Frantz Fanon et Ernesto Che Guevara, et de la résolution du Congrès cubain pour l'éducation et la culture d'avril 1971. On y discuta de la nécessité de dépasser et de rompre le mythe de l'eurocentrisme colonisant et l'application schématique de la pratique socialiste européenne, qui ne correspond presque jamais à la réalité socio-économique culturelle latinoaméricaine. Et toutes les analyses techniques s'inscrivirent dans ce contexte ${ }^{60}$.

Dans le contexte de la Guerre froide et de ses conflits périphériques, certains leaders politiques du tiers-monde, tels Fidel Castro, Mao Zedong et Ernesto «Che » Guevara, représenteraient «tout à la fois la lutte révolutionnaire contre l'Amérique [les ÉtatsUnis], le changement social radical et, pour les deux derniers, une remise en cause de la norme soviétique ${ }^{61} »$. Le choix d'utiliser les discours de Castro, de Guevara et de Lumumba dans Für Paul Dessau suit cette logique de décentrement et d'internationalisation des luttes d'émancipation anti-impérialistes ${ }^{62}$. Ces figures politiques s'engageaient dans un combat idéologique contre la vision bipolaire du monde et voulaient donner aux nations périphériques un rôle historique à jouer. $\mathrm{Ce}$ rôle n'était pas seulement politique mais aussi idéologique et culturel, en montrant une voie différente à suivre aux sociétés qui refusaient de se soumettre à la logique géopolitique des deux blocs. De ce fait, comme le souligne Robert Frank, « la nouvelle multipolarité n'est donc pas seulement une affaire géopolitique de rapports de force internationaux, comme à la fin des années 1950 ; elle se situe désormais à partir du milieu des années 1960 au cœur d'une grille de lecture idéologique, politique et culturelle ${ }^{63} »$.

La dimension idéologique et historique de Für Paul Dessau suit cette logique. Par la synthèse et la spatialisation des discours et des sons utilisés, l'œuvre matérialise le tiers-monde dans un espace symbolique unifié et ancré dans une tradition socialiste, rendant possible sa mobilisation comme un concept opératoire pour penser l'unité des luttes postcoloniales des pays qui s'y identifiaient. Au tournant des années 1970, cette volonté de donner corps à l'idée du tiers-monde se plaçait au cœur de la lutte idéologique des grands projets politiques, à l'encontre de l'opinion de certains intellectuels - comme Hannah Arendt, qui n'hésitait pas à qualifier le tiers-monde de n'être qu'une « illusion ${ }^{64} »$. Dans cette confrontation idéologique si caractéristique de la Guerre froide, qui consistait à tenter d'imposer dans l'espace public des concepts et des représentations tout en les opposant à ceux des adversaires, Für Paul Dessau constitue le réquisitoire de Luigi Nono contre l'« Occident impérialiste", symbolisé avant tout par les États-Unis et leur politique en Amérique latine, en Afrique et en Asie. La dimension politique de l'œuvre de Nono peut être analysée comme une stratégie consciente pour l'hégémonie intellectuelle et culturelle socialiste dans sa dimension propagandiste,

Transposition, 4 | 2014 
c'est-à-dire en tant que stratégie de légitimation «qui viserait non seulement à influencer mais aussi provoquer l'identification et l'adhésion consciente des individus à un pouvoir perçu comme légitime ${ }^{65}$ ». Dans Für Paul Dessau, cette stratégie consisterait notamment à donner la parole à des acteurs spécifiques en les rendant visibles dans l'espace commun, à légitimer des idées et des concepts pour penser le réel ${ }^{66}$. Comme le soutient Nono,

si une partition ne peut pas provoquer ou susciter une révolution, elle peut y contribuer, en participant à l'hégémonie intellectuelle et révolutionnaire. Une partition peut mûrir et se résoudre dans la participation directe et concrète de la lutte, qui peut être affrontée et transposée dans la partition. La musique, comme intervention active, manifestation et expression d'une pensée, d'une méthode, d'une analyse et d'un choix humain, pour laquelle le moyen technique n'est pas un instrument de simple modernisation, mais la voix du peuple ${ }^{67}$.

Grâce aux moyens offerts par l'électronique, Für Paul Dessau "transpose » et relie symboliquement les voix d'un archipel de luttes politiques et de conflits armés du XX siècle, esquissant une cartographie des luttes du tiers-monde - comme celle énoncée par Guevara dans son discours - à l'intérieur d'une généalogie des luttes historiques internationales. L'œuvre, dans sa matérialité, apparaît ainsi comme une mémoire sonore du rôle de l'utopie socialiste dans les luttes d'émancipation et la trace de l'espoir soulevé par de cette utopie chez Luigi Nono.

\section{Conclusion}

Dans Für Paul Dessau, Nono crée un document sonore, à la fois personnel et historique, qui témoigne du chemin politique et esthétique qu'il a parcouru en compagnie d'autres intellectuels et artistes du XX ${ }^{\mathrm{e}}$ siècle - dont Paul Dessau - ainsi que des luttes politiques d'émancipation des peuples du tiers-monde. Cette œuvre, ainsi que les œuvres qui sont liées à elle d'une manière ou d'une autre, répond à la problématique concrète de la politisation de la musique durant la Guerre froide: l'utilisation stratégique de la création musicale et de la technologie au service d'un idéal politique. Cependant, le rapport de Für Paul Dessau avec les conflits armés n'est pas direct et fonctionnel car elle n'a pas été conçue expressément, par exemple, pour être jouée au front, pour inciter ou pour faire cesser la violence pendant ou après des opérations militaires, pour témoigner des atrocités de la guerre ou pour être écoutée par les populations civiles subissant directement la destruction provoquée par celle-ci. L'œuvre n'a pas été composée non plus dans une situation de guerre car l'utilisation des ressources matérielles et techniques nécessaires à sa conception aurait été extrêmement difficile.

$\mathrm{Au}$ contraire, Für Paul Dessau est conçue pour territorialiser symboliquement des conflits et des luttes politiques éloignées au premier abord, pour relier des idéologies politiques transmises lors de discours historiques afin de rendre concret un horizon de lutte et d'émancipation, soutenu par un idéal politique et par l'espoir de dépasser ces conflits. Comme l'explique Helmut Lachenmann, «la volonté qu'avait Nono d'exercer un effet politique - parallèlement ou en travers de la doctrine de son parti - est un élément indispensable de sa folie "prométhéenne", de sa volonté "d'apporter le feu" à l'humanité, au risque d'être lui-même châtié par ces puissances ${ }^{68}$ ». Se concevant lui même comme un musicien militant "non au-dessus mais dans la lutte des classes telle qu'elle existe - "intellectuel faisant partie de la classe ouvrière", selon le vœu d'Antonio Gramsci ${ }^{69}$ ", Luigi Nono a cherché à associer militantisme politique et création 
symbolique afin de remplir la fonction hégémonique qu'il accordait aux intellectuels organiques. Il entend ainsi prendre sa responsabilité en tant qu'artiste dans les processus de transformation culturelle, politique et sociale de son époque : «dans cette nécessité de plus en plus impérieuse d'une liaison internationale efficace des forces de paix ou d'action révolutionnaire, il me semble que l'artiste a un rôle important à jouer ${ }^{70}$ $»$.

En saisissant les nouvelles possibilités offertes par les moyens électroacoustiques à la composition musicale, notamment leur capacité à transformer des sons en les insérant dans un nouvel espace acoustique ainsi qu'à figer et à transmettre des témoignages, Nono donne la parole aux acteurs du passé et de son époque. Ceci confère à Für Paul Dessau - de même qu'à une grande partie de ses œuvres de cette période - une profondeur historique capable d'affronter le passage du temps: une mémoire contre l'oubli. Ainsi, Nono accomplit le but que William Faulkner, dans un entretien datant de 1956, accorde à l'artiste :

Le but de l'artiste est d'arrêter le mouvement, qui est la vie, par des moyens artificiels et de le maintenir figé pour que cent ans plus tard, quand un inconnu le regardera, il se remette à bouger puisque c'est la vie. Comme l'homme est mortel, la seule immortalité possible pour lui est de laisser derrière lui quelque chose d'immortel puisque cela bougera toujours ${ }^{71}$.

Dans Für Paul Dessau, Luigi Nono tente d'arrêter le temps en laissant une œuvre qui témoigne des luttes communes de nombreux intellectuels dans un moment où l'espoir dans un monde renouvelé venait de la conjonction entre les combats de libération du tiers-monde et ceux des intellectuels, des étudiants et des ouvriers des pays développés. Néanmoins, étant donné les défaites de ces idéaux et les dérives des certains de ces projets révolutionnaires - de Cuba à la Chine, en passant par la Russie il est important de replacer Für Paul Dessau dans son contexte de création afin saisir toute sa complexité et son originalité. En ce sens, l'œuvre de Nono porte la trace des grands conflits de son époque, tant politiques qu'humains, qui se trouvaient au centre de ses préoccupations et de son activité créatrice, comme il le déclarait en 1988 :

Ce qui me passionne vraiment, ce qui me fascine, ce sont les grands conflits humains, les grands affrontements, comme la révolution cubaine, le drame de Galilée, celui de Giordano Bruno, le drame de Gramsci, celui de Hölderlin, le drame de ceux qui se sont suicidés: Jackson Pollock, qui a connu une fin dramatique, Maïakovski... Je crois que ce qui m'a toujours passionné est le moment du conflit ${ }^{72}$.

\section{NOTES}

1. NONO, Luigi, "Entretien avec Luigi Nono", in Écrits, textes édités et traduits par Laurent FENEYROU, Genève, Contrechamps, 2007, p. 301.

2. D'après Nono, «les ouvriers imposent, dans l'usine de classe, une lutte pour le pouvoir socialiste. Les guérilleros imposent un conflit de classe, frontal. (Le second - le conflit ou la lutte armée - est incontestablement le moment décisif.) », Idem., p. 302-303.

3. Comme il l'écrit en 1966, pour lui « il n'y a pas de différence entre la lutte de classes et celle des peuples pour leur libération », NONO, Luigi, « Un discours sonore », in Écrits, op. cit., p. 233. 
4. L'œuvre est disponible à l'écoute ici : https://www.youtube.com/watch?v=CnGwMPLKYjU

5. Le catalogue d'œuvres pour bande magnétique seule de Luigi Nono est composé par Omaggio a Emilio Vedova (1960), Musiche di scena per Die Ermittlung (1965), Ricorda cosa ti hanno fatto in Auschwitz (1966), Contrappunto dialettico alla mente (1967-1968), Musiche per Manzù (1969) et Für Paul Dessau (1974) - voir NONO, Luigi, Complete Works for Solo Tape (2 CD + livret), Stradivarius Ricordi Oggi (STR 57001), 2006.

6. D'une durée de sept minutes, Für Paul Dessau est créée le 15 décembre 1974 à l'Académie des Arts de Berlin-Est lors d'un concert-hommage à Dessau.

7. Notamment, par ordre chronologique, Il canto sospeso (1955-1956), La fabbrica illuminata (1964), A floresta é jovem e cheja de vida (1966), Non consumiamo Marx (1969), Y entonces comprendió (1970), Ein Gespenst geht um in der Welt (1971), Como una ola de fuerza y luz (1971-1972) et Al gran sole carico d'amore (1972-1974). Pour un aperçu complet du catalogue et des notices des œuvres de Luigi Nono voir NONO, Luigi, Écrits, op. cit., p. 593- 691.

8. NONO, Luigi, « Le pouvoir musical », in Écrits, op. cit., p. 321.

9. Le choix d'utiliser ces notions développées par Gramsci se justifie par l'influence de celui-ci sur la pensée politique de Nono, qui, comme nous le verrons tout au long de cet article, le cite constamment et le montre comme une référence incontournable.

10. GRAMSCI, Antonio, « Cahier 11 », in Cahiers de prison, t. 3, Paris, Gallimard, 1978, p. 180.

11. NONO, Luigi, « Un discours sonore », art. cit., p. 233-234.

12. Idem.

13. Pour Gramsci « tous les hommes sont intellectuels ; mais tous les hommes ne remplissent pas dans la société la fonction d'intellectuels » (GRAMSCI, Antonio, "Cahier 12 », in Cahiers de prison, t. 3, op. cit., p. 312). Il différencie donc deux classes d'intellectuels : les intellectuels organiques, reliés " organiquement » à une classe sociale et définis par la place et la fonction qu'ils occupent dans la structure sociale (par exemple, les cadres de l'appareil politique, culturel ou judiciaire dans leur rapport à la bourgeoisie ou au prolétariat) ; et les intellectuels traditionnels, reliés à des classes disparues ou en voie de disparition et définis par la place et la fonction qu'ils occupent au sein d'un processus historique (par exemple, le clergé dans son rapport à l'aristocratie). Sur ce sujet, voir PIOTTE, Jean-Marc, La pensée politique de Gramsci, Montréal, Lux, 2010 [1970], p. 17-66.

14. NONO, Luigi, « Le pouvoir musical », art. cit., p. 319.

15. Idem.

16. Idem., p. 317.

17. Le travail de Stockhausen au Studio de musique électronique de la radio de Cologne (Westdeutscher Rundfunk - WDR) comportait en 1969 un certain nombre d'œuvres électroniques ou mixtes, notamment Gesang der Jünglinge (1956), Kontakte (1960), Mixtur (1964), Mikrophonie I (1964), Mikrophonie II (1965), Telemusik (1966) et Hymnen (1966-1967).

18. Nono parle de cette œuvre dans un entretien publié en janvier 1969 dans la revue vénézuélienne Rocinante: «Un compositeur de l'Allemagne de l'Ouest, Stockhausen, qui milite pour l'électronique, jusqu'à la polémique et à des conflits personnels violents, a ressenti le besoin d'utiliser pour l'une de ses compositions, Hymnen, des matériaux acoustiques localisées historiquement et géographiquement, des hymnes nationaux qu'il traite avec "objectivité" et avec un certain "sentiment de supériorité" (l'hymne espagnol du fasciste Franco avec celui de la République populaire d'Albanie ou le chant communiste italien Bandiera rossa avec un chant nazi...)», NONO, Luigi, «Entretien avec Luigi Nono», art.cit., p. 298. Pour une description analytique de la première version de Hymnen voir HARVEY, Jonathan, «Stockhausen's Hymnen », in Musical Times 116, no. 1590, 1975, p. 705-707.

19. NONO, Luigi, « Le pouvoir musical », art. cit., p. 318.

20. Les intellectuels seraient porteurs pour Gramsci de la fonction hégémonique de la classe à laquelle ils appartiennent : «ils travaillent dans les différentes organisations culturelles [...] de façon à assurer le consentement passif sinon actif des classes dominées à la direction qu'imprime 
à la société la classe dominante. [...] Par les moyens de diffusion qu'il emploie et par le rôle d'éducateur de ses militants, le prolétariat se dresse comme adversaire de l'hégémonie qu'exerce la bourgeoisie, et tend à la renverser », (PIOTTE, Jean-Marc, La pensée politique de Gramsci, op. cit., p. 22).

21. LACHENMANN, Helmut, "Touché par Nono » (1991), in Écrits et entretiens, textes choisis et préfacés par Matin KALTENECKER, Genève, Contrechamps, 2009, p. 184.

22. FENEYROU, Laurent, « Il Canto sospeso » de Luigi Nono, Paris, Michel de Maule, 2002, p. 112-113.

23. Pour Gramsci, «créer une nouvelle culture ne signifie pas seulement faire individuellement des découvertes "originales", cela signifie aussi, et spécialement, répandre de façon critique les découvertes déjà faites, les "socialiser" pour ainsi dire, et par conséquent faire qu'elles deviennent autant de bases pour des actions vitales, en faire un élément de coordination et d'ordre intellectuel et moral », GRAMSCI, Antonio, "Cahier 11 », in Cahiers de prison, t. 3, op. cit., p. 177.

24. NONO, Luigi, «Entretien avec Luigi Nono », art.cit., p. 297.

25. Les discours sont en russe, allemand, français et espagnol. La plupart de ces disques vinyles se trouvent actuellement à Venise dans les archives Luigi Nono (http://www.luiginono.it).

26. Paul Dessau écrit son Requiem für Lumumba (pour soprano, baryton, récitant, chœur et orchestre) en 1963 et son œuvre pour orchestre et chœur Lenin en 1969. À propos de son amitié avec Dessau, Nono témoignait en 1973 : «L'amitié qui me lie à lui est très importante pour moi parce que, en dehors de l'admiration que je porte à ses contributions artistiques profondément humaines et engagées politiquement, nous sommes très proches d'un point de vue politique » [L'amicizia che mi lega a lui è per me molto importante poiché, a parte l'ammirazione che porto alle sue opere artistiche profondamente umanitarie e politicamente impegnate, mi è anche da un punto di vista politico molto vicino] («Intervista di Jean Villain» (1973), in NONO, Luigi, Scritti e colloqui, édité par Angela Ida DE BENEDICTIS et Veniero RIZZARDI, vol. II, Milan, 2001, p. 141 ; cité dans NONO, Luigi, Complete Works for Solo Tape (2 CD + livret), op. cit., p. 34). Sauf mention contraire, toutes les traductions de l'espagnol et de l'italien au français ont été réalisées par mes soins.

27. LACHENMANN, Helmut, « Touché par Nono », art. cit., p. 184.

28. NONO, Luigi, Écrits, op. cit., p. 661.

29. Non consumiamo Marx constitue la deuxième partie de Musica-Manifesto $n$. 1, dont la première partie, Un volto, e del mare, est écrite pour deux voix de femme et bande magnétique.

30. Pour une analyse approfondie de cette œuvre voir FENEYROU, Laurent, « Il Canto sospeso » de Luigi Nono, op. cit.

31. NONO, Luigi, Écrits, op. cit., p. 604.

32. FENEYROU, Laurent, « Il Canto sospeso » de Luigi Nono, op. cit., p. 14.

33. NONO, Luigi, Écrits, op. cit., p. 648.

34. «Movimiento de izquierda revolucionaria ».

35. NONO, Luigi, Écrits, op. cit., p. 657-658.

36. « ...addio mamma, tua figlia Ljubka se ne va nell'umida terra... » (reproduit dans FENEYROU, Laurent, «Il Canto sospeso » de Luigi Nono, op. cit., p. 205). Ljuba Sevcova sera torturée puis achevée d'une balle dans la tête le 7 février 1943 par un Rottenführer des SS (voir Idem., p. 213-214).

37. NONO, Luigi, « Musique et Resistenza », in Écrits, op. cit., p. 166-167.

38. Idem., p. 167.

39. Idem.

40. Comme le note Erika Schaller, Nono a conçu la version stéréo (utilisée lors de la création de l'œuvre à Berlin) à partir d'une version pour 4 pistes (SCHALLER, Erika, «Für Paul Dessau », in NONO, Luigi, Complete Works for Solo Tape (2 CD + livret), op. cit., p. 64).

41. Dans ses réflexions stratégiques sur la guerre de mouvement et la guerre de position, Gramsci place la Russie dans un contexte "oriental », sur ce sujet voir GRAMSCI, Antonio, Guerre de 
mouvement, guerre de position, textes choisis et présentés par Razmig Keucheyan, Paris, La Fabrique, 2011, p. 35-44.

42. Sur l'importance historique de la Conférence Tricontinentale voir FALIGOT, Roger, Tricontinentale. Quand Che Guevara, Ben Barka, Cabral, Castro et Hô Chi Minh préparaient la révolution mondiale (1964-1968), Paris, La Découverte, 2013.

43. NONO, Luigi, Carteggi concernenti politica, cultura et partito comunista italiano, édité par Antonio TRUDU, Vénice, Leo S. Olschki (Archivio Luigi Nono studi), 2008, p. 199.

44. SCHALLER, Erika, «Für Paul Dessau», art.cit, p. 65. L'auteure fait référence à un brouillon (non daté) du programme du concert-hommage à Dessau qui se trouve dans les archives Luigi Nono.

45. "Qu'est-ce que le pouvoir des Soviets ?», prononcé fin mars 1919 et publié dans la Pravda le 21 janvier 1928, réproduit dans LÉNINE, Vladimir, Oeuvres, t. 29, Éditions sociales, Paris, 1962, p. 250-251; «Adresse à l'armée rouge », prononcé le 29 mars 1919 et publié dans le même ouvrage, p. 246-247. Le disque vinyle édité à Rome par Editori Riuniti (avec la traduction italienne des discours) se trouve dans les archives Luigi Nono (voir http://www.luiginono.it/it/node/17853). Nous pouvons également apercevoir l'influence de Gramsci à travers l'importance que Nono donne à Lénine dans sa pensée politique : pour Gramsci, le leader bolchevique constituait «le modèle de l'intellectuel intégral » (PIOTTE, Jean-Marc, La pensée politique de Gramsci, op. cit., p. 37). 46. LÉNINE, Vladimir, "Qu'est-ce que le pouvoir des Soviets?», art. cit., p. 251. Je citerai exclusivement des extraits des parties des discours utilisées par Nono dans Für Paul Dessau.

47. LÉNINE, Vladimir, « Adresse à l'armée rouge », art. cit., p. 247.

48. NONO, Luigi, «Intervention contre la guerre du Viêt-Nam» (1966), in Écrits, op. cit., p. 251. Les extraits entre crochets sont des ajouts manuscrits (mais non de la main de Nono), sur le tapuscrit original, (voir Idem., p. 252, note 4 et sq.). La Sierra Maestra est le massif montagneux situé à l'Est de l'île de Cuba, qui a servi de refuge et de quartier général aux troupes commandées par Fidel Castro durant la Révolution cubaine.

49. «América, Asia y África, los tres continentes oprimidos estaban dando señales de que no admitirían por mucho tiempo más la presencia de los poderes coloniales », in GUEVARA, Ernesto "Che », Solidaridad con Vietnam del Sur - Discurso del Comandante Ernesto Guevara en el Acto de Clausura de la Semana de Solidaridad con el Pueblo de Viet-Nam del Sur, 20 décembre 1963 (disque vinyle EGREM-CNC LD-DP-7). Le disque vinyle se trouve dans les archives Luigi Nono (voir http://www.luiginono.it/it/node/17818).

50. "Se tendrá que contar con los pobres de América, con los explotados y vilipendiados de América latina que han decido empezar a escribir ellos mismos, para siempre, su historia ", in CASTRO, Fidel, Segunda declaración de La Habana, Instituto cubano de amistad con los pueblos (discours prononcé à La Havane, à la Place de la Révolution, le 4 février 1962). Le disque vinyle se trouve dans les archives Luigi Nono (voir http://www.luiginono.it/it/node/17813).

51. «Cuba, por los alfabetizadores asesinados; Estados Unidos, por los asesinos / Cuba, por la verdad; Estados Unidos, por la mentira / Cuba, por la liberación; Estados Unidos, por la opresión / Cuba, por el porvenir luminoso de la humanidad; Estados Unidos, por el pasado sin esperanza / Cuba por la paz entre los pueblos; Estados Unidos por la agresión y la guerra / Cuba, por el socialismo, Estados Unidos, por el capitalismo ", in CASTRO, Fidel, Segunda declaración de La Habana, op. cit.

52. NONO, Luigi, «Intervention contre la guerre du Viêt-Nam » (1966), in Écrits, op. cit., p. 250.

53. Lumumba est assassiné en prison au Katanga, en janvier 1961 à l'âge de 35 ans, avec la complicité des États-Unis et de la Belgique.

54. Le disque vinyle « Ultimo discorso di Patrice Lumumba » produit par la maison Italia Canta se trouve dans les archives Luigi Nono (voir http://www.luiginono.it/it/node/17846).

55. LUMUMBA, Patrice, « Ultimo discorso di Patrice Lumumba ». 
56. Ernst Thälmann (1886-1944), l'un des dirigeants du Parti communiste d'Allemagne (KPD), siège au Reichstag de 1924 à 1933 avant d'être arrêté par le pouvoir nazi. Incarcéré à la prison d'État de Bautzen, il est transféré au camp de concentration de Buchenwald où il est assassiné le 18 août 1944.

57. «Die Voraussetzungen für die großen Aufgaben des Sieges der proletarischen Revolution zu erfüllen. Und in diesem Sinne überbringe ich dem Kongreß die brüderlichsten und die revolutionärsten Grüße », Ernst Thälmann, discours prononcé à Moscou le 15 février 1928, dans le cadre du Congrès des travailleurs de la métallurgie (pour l'audio, voir https://archive.org/ details/SelectedWeimarEraPoliticalSpeechesPart2, consulté le 28 mars 2014, traduit de l'allemand par Stéphanie Gernet).

58. Journal fondé par Antonio Gramsci en 1924, il a été l'organe officiel du Parti communiste italien (PCI) jusqu'à la dissolution de celui-ci en 1991.

59. "Quel socialismo alla cui realizzazione tendiamo, creandolo nella nostra realtà, che abbiamo imparato da Lenin da Gramsci dal soviettismo dalle conduzioni delle lotte di liberazione in Asia [...], in Africa e in parte dell'America latina, che stiamo costruendo con la lotta degli operai dei contadini dei braccianti degli studenti, quel socialismo per cui lottano gli operai della regione baltica, fino a quelli di Detroit e al movimento dei Black Panthers nella roccaforte dell'imperialismo », NONO, Luigi, «Luigi Nono a "L'Unità" [Venezia, gennaio 1971] », in NONO, Luigi, Carteggi concernenti politica, cultura et partito comunista italiano, op.cit., p. 178.

60. NONO, Luigi, « Cours latino-américain de musique contemporaine », in Écrits, op. cit., p. 369.

61. FRANK, Robert, «Imaginaires politiques et figures symboliques internationales : Castro, Hô, Mao et le "Che" ", in DREYFUS-ARMAND, Geneviève et al. (éd.), Les années 68. Le temps de la contestation, Bruxelles, Complexe, 2000, p. 35.

62. Par ailleurs, Nono utilise des citations des textes de Gramsci, Fanon, Castro, Guevara et Lumumba, dans certaines des œuvres composées à la même période que Für Paul Dessau, notamment dans A floresta é jovem e cheja de vida (1966, textes de Fidel Castro, Frantz Fanon, Herman Kahn et Patrice Lumumba), Y entonces comprendió (1970, textes de Ernesto "Che » Guevara et Carlos Franqui), Ein Gespenst geht um in der Welt (1971, textes de Karl Marx, Celia Sánchez et Haydée Santamaría) et Al gran sole carico d'amore (1972-1974, textes de Bertolt Brecht, Tania Bunke, Fidel Castro, Ernesto "Che» Guevara, Georgi Dimitrov, Maxime Gorki, Antonio Gramsci, Lénine, Karl Marx, Louise Michel, Cesare Pavese, Arthur Rimbaud, Celia Sánchez et Haydée Santamaría).

63. FRANK, Robert, «Imaginaires politiques et figures symboliques internationales : Castro, Hô, Mao et le "Che" ", art. cit., p. 36.

64. ARENDT, Hannah, "Sur la violence», in Du mensonge à la violence. Essais de politique contemporaine [1972], Paris, Gallimard, 2012, coll. Quarto, p. 993. Hannah Arendt oppose au caractère «irréel » et «illusoire » du tiers monde le caractère «réel » de "l'Afrique, l'Asie et l'Amérique du Sud», sans pour autant questionner ou déconstruire la dimension historique de ces notions. Pour une discussion sur ce sujet dans le contexte des luttes d'émancipation afroaméricaines des années 1960 voir LOSURDO, Domenico, La non-violenza: una storia fuori dal mito, Roma, Laterza, 2010, p. 154-156.

65. VELASCO-PUFLEAU, Luis, "Réflexions sus les rapports entre Musique et Propagande ", in Music and Propaganda in the Short Twentieth Century, Massimiliano Sala (ed.), Turnhout, Brepols, 2014, p. 5.

66. Ceci rejoint la vision que Jacques Rancière à propos du «partage du sensible " et de la capacité des pratiques de l'art à «contribuent à dessiner un paysage nouveau du visible, du dicible et du faisable » (RANCIÈRE, Jacques, «Les paradoxes de l'art politique », in Le spectateur émancipé, Paris, La Fabrique, 2008, p. 84).

67. NONO, Luigi, «Entretien avec Luigi Nono », art.cit., p. 301.

68. LACHENMANN, Helmut, « Touché par Nono ", art. cit., p. 184. 
69. NONO, Luigi, «Une lettre de Luigi Nono : "Je suis un musicien militant" ", in Écrits, op. cit., p. 347

70. NONO, Luigi, « Un discours sonore », art.cit., p. 233.

71. FAULKNER, William, «The Art of Fiction No. 12 » (interviewed by Jean Stein), Paris Review, $\mathrm{n}^{\circ}$ 12, Spring 1956, (texte intégral en anglais sur http://www.theparisreview.org/interviews/4954/ the-art-of-fiction-no-12-william-faulkner, consulté le 9 juin 2014). Traduction française in Paris Review. Les Entretiens, Vol. 2, traduit par Anne Wicke, Paris, Christian Bourgois, 2011, p. 236.

72. Extrait du film documentaire Archipel Luigi Nono, produit et réalisé par Olivier Mille, Artline Films/ La Sept, 1988 (37'27"-38'04”). Je remercie Joséphine Markovits de m'avoir fait connaître l'existence de ce document.

\section{RÉSUMÉS}

Au-delà de l'horreur que provoque la guerre en elle-même, certains conflits armés du XX $\mathrm{XX}^{\mathrm{e}}$ siècle ont suscité un grand espoir chez des artistes marxistes engagés dans les luttes politiques internationales de leur époque. Ce fut notamment le cas du compositeur italien Luigi Nono (1924-1990). Je propose d'examiner dans cet article de quelle façon Nono place certains des conflits armés, dont les luttes d'émancipation du tiers-monde, au cœur de son œuvre pour bande magnétique Für Paul Dessau (1974). De même, j'analyse la relation entre l'utilisation de la technologie par le compositeur, la pensée politique de ce dernier et les dimensions historique et esthétique de l'œuvre - notamment dans le contexte de la Guerre froide et de l'idéologie marxiste. Plus largement, l'ambition de ce texte est de contribuer à la compréhension et à la mise en perspective du marxisme défendu par Luigi Nono en tant qu'intellectuel et artiste militant au Parti Communiste Italien (PCI), dans son articulation avec ses idées esthétiques et son activité de compositeur.

Despite the horror caused by war, some armed conflicts of the twentieth century have raised great hopes for social justice among Marxist artists engaged in the international political struggles of their time. Such was the case of the Italian composer Luigi Nono (1924-1990). In this article, I examine how Nono addresses some of these armed conflicts, such as Third World liberation struggles, through his work for magnetic tape Für Paul Dessau (1974). I also analyse the relationship between the composer's use of technology, his political thinking and the historical and aesthetic dimensions of this work - especially within the context of the Cold War and the Marxist ideology. More broadly, this paper aims at contributing to the understanding of Marxism as advocated by Luigi Nono, as an intellectual and an activist artist in the Italian Communist Party (PCI), so as to see how it is articulated with his aesthetics and compositional practice.

\section{INDEX}

Mots-clés : Luigi Nono, Paul Dessau, musique électroacoustique, conflits armés, marxisme, idéologie, hégémonie

Keywords : Luigi Nono, Paul Dessau, electroacoustic music, armed conflicts, Marxism, ideology, hegemony 


\section{AUTEUR}

\section{LUIS VELASCO-PUFLEAU}

Docteur en Musique et musicologie (Université Paris-Sorbonne) et chercheur post-doctorant à l'Université de Salzbourg, Luis Velasco-Pufleau a été également chercheur post-doctorant au Centre de recherches sur les arts et le langage (CRAL) de l'École des hautes études en sciences sociales (EHESS, Paris) et chargé de cours en musicologie à l'Université de Bordeaux. Ses recherches portent sur la création musicale contemporaine ainsi que sur les rapports entre musique, esthétique et politique au $\mathrm{XX}^{\mathrm{e}}$ siècle. 